\title{
A Tentative Exploration on Multimodal Teaching of Legal English*
}

\author{
Yuehua Lu \\ School of Foreign Languages and Cultures, Shanghai University of Political Science and Law, China
}

\begin{abstract}
Based on the theory of multimodality and Internet technology, the present paper analyzes the existing problems in legal English teaching, explores the feasibility of constructing a multimodal teaching mode, and presents a case study of moot court by applying the multimodal means of teaching. The tentative study proves that a multimodal teaching mode can satisfy the requirements of legal English teaching, though there still exist problems to be solved.
\end{abstract}

Index Terms - legal English, multimodality, moot court, ESP teaching

\section{INTRODUCTION}

In the 1990s, with the rapid development of multimedia technology and corpus research, multimodal discourse analysis, as a new theory of discourse analysis, has been incorporated into language research. This research focuses on the realization of meaning, holding that not only language, but also all other symbol systems have realized meaning. This theory exceeds the limitation of traditional language analysis which studies language itself in isolation, extends the research to other modal symbols such as image, color and font, and studies the role of various modal symbols in classroom discourse.

The year 2020 witnessed the spread of COVID-19 throughout the world. To enable the students who are stuck at home to continue their study and complete the corresponding teaching process, online teaching, a new teaching form has become the dominant way of education at this stage. Although online teaching is an expedient, its advantages have been found and recognized by more teachers as well as students. Looking into the future, the combination of online and offline teaching will become an irreversible trend. Hybrid teaching is not only the teaching of mixed resources, but also the teaching of mixed multi-modal environment. In the process of teachers' teaching and students' learning, by employing video, audio, picture, text and other multimedia devices, a multi-modal teaching environment integrating vision, hearing, speaking, and gesture is thus created, which will improve the learning efficiency and effect.

Legal English, taking common English as its basis, is a language with legal characteristics formed and used in legislation, judicature and other legal-related activities. Legal English is normally used to express legal scientific concepts, and it is employed either in litigation or non-litigation legal affairs. At present, the legal English course has become part of the curricula in most law schools or foreign language schools in China's political and Law Universities and some comprehensive universities. The construction of legal English course is an effective means to realize the goal of cultivating legal talents.

The present paper, by referring to the theory of multimodality and Internet technology, is intended to explore the feasibility and then construct a multimodal teaching mode of the legal English course.

\section{THE THEORY OF MuLTIMODALITY}

\section{A. The Theory of Multimodality}

Modality is defined as the way in which human beings interact with the external environment through their senses (Gu, 2007). It is the meaning potential formed by material media after a long time of social shaping, and it is a social and cultural resource used to represent and exchange meaning. Undoubtedly, language is the most common mode. However, such items as image, intonation, sound, expression, gesture, color, video, Internet, laboratory, PPT and other modern technological devices can all be called mode. The five communication modes, namely, visual mode, auditory mode, tactile mode, olfactory mode and gustatory mode arise from the five senses of human beings (Zhu, 2007).

Originated from Functional Linguistics and Social Semiotics in 1990s in the West, multimodal research has achieved rapid development. The most outstanding researchers, such as Kress (2001), Royce (2002), and Jewitt (2016), to name just a few, have conducted systematic explorations on the multimodal phenomena in different social and cultural fields, including architecture, painting, sculpture, mathematics, language and so on. Over more than 20 years of development, multimodal research has far transcended the field of linguistics, extending to semiotics, philosophy, psychology, sociology, anthropology, politics, journalism, law, aesthetics, and medicine. The research objects have also expanded

\footnotetext{
* This paper is part of the results of the scientific research project entitled Building a Multimodal Teaching Mode of Legal English under the Background of Internet of Shanghai University of Political Science and Law. The project number is 2020 XJ19.
} 
from language to music, image, web design and architectural style.

In China, although multimodal research started late, it develops rapidly. Li Zhanzi $(2003,2012)$ introduced the theory of multimodal discourse analysis from the perspective of social semiotics, which initiates the study of multimodality. Since then, Hu Zhuanglin (2007), Zhu Yongsheng (2007), Zhang Delu (2009, 2010, 2012, 2019) and other scholars have studied the relationship between multimodality and computer semiotics, the theoretical basis and methods of multimedia, multimodal learning analysis, and multimodal discourse analysis, all of which have played a macro guiding role in promoting domestic multimodal research.

\section{B. Multimodal Teaching in China}

Modern teaching itself, characterized by multimedia, online courses and other means of modern education, is multimodal. Under the circumstance of multimedia and network information teaching, modality has become a means as well as a method for teachers and students to acquire, recognize and transmit information via various senses. The relationship between media and modality can be interpreted as that between entity and form (Zhang, 2019). Different media entities are the concrete embodiment of different modality forms. All the tools employed in multimedia teaching such as text, picture, video, and PPT can be regarded as modes. The coordination of these multimedia teaching aids is actually a multi-modal synergy, in which words, pictures, teachers' gestures and facial expressions can be regarded as visual modes, and teachers' explanation and various audio files are auditory modes. In addition, the network contains both the auditory mode and the visual mode, which are the most important and frequently used modes in teaching. Applying multiple modes will definitely achieve better effects than using only one mode, and the best result can be achieved by using various modes flexibly and giving full play to the positive synergy of different modes.

Professor Zhang Delu from Tongji University is the leading representative of scholars engaged in multimodal teaching research. Prominent scholars who have published papers in CSSCI journals also include Huang Lihe (2019), Cheng Ruilan (2017), Liu Xiuli (2013) and others. Multimodal teaching integrates text language elements and non-language elements such as vision, hearing, expression, action and gesture, empathizing the synergetic effect of multi senses and multi modes. The realization of multimodal teaching depends on the change of teachers' concept of education, which requires teachers to design multimodal tasks as much as possible, and guide students to comprehensively use multimodality to fulfill the tasks. There is no doubt that the effect of using multimodal teaching method is better than that of using only one mode, which will help students to receive and digest what they have learned in class.

\section{LEGAL ENGLISH TEACHING IN CHINA}

\section{A. An Overview of Legal English}

Legal English, one of the most important branches of English for specific purposes (ESP), is a new interdisciplinary subject of legal science and English linguistics. With the gradual integration of foreign language education and legal education, legal English has been established as a professional direction in many colleges and universities, especially in political and legal ones. Legal English is affiliated to legal linguistics, and it is by no means a simple combination of law and English (Zhang, 2019). To master legal English, one must have both legal and English backgrounds. Specifically speaking, the course of legal English, based on the knowledge of common law, systematically teaches the Anglo-American legal system, including British and American legal culture, major department laws, as well as the corresponding legal English translation, legal English writing, case analysis and foreign-related practical skills. With the development of legal English, there appeared a considerable number of scholars who are devoted to the study of legal English, such as Zhang Falian (2009, 2013, 2019), Qu Wensheng (2017), Ma Qinglin (2019) and others, who focus their research mainly on the cognition of the characteristics of legal language, namely, vocabulary, terminology, syntax, the translation of legal terms, legal texts, and laws and regulations.

\section{B. Present Situation of Legal English Teaching in China}

Legal English teaching, which first appeared in the late 1970s and early 1980s, has achieved rapid development since the beginning of the $21^{\text {st }}$ century. The teaching objective is to cultivate applied talents with proficient English language skills and certain basic knowledge of law, strong cross-cultural communication ability, broad international vision and certain practical and innovative ability, who can deal with foreign legal affairs and engage in relevant interpretation and translation. However, legal English is different from ordinary college English and traditional English courses in that it belongs to the category of English for Specific Purposes (ESP). To put it another way, legal English is related directly to law, explaining legal problems and teaching legal knowledge in English, aiming to impart the knowledge of British and American legal culture and legal system. Secondly, the nature of ESP leads to the second feature of legal English teaching, that is, content-based teaching, which means that language teaching is built on the teaching of certain subject content and the language materials are authentic. According to the concept of content-based teaching, only when learners regard language as a means rather than a purpose of learning subject knowledge and obtaining information, only when learners feel that the information that they obtain is useful, interesting and satisfying, and only when teaching is aimed at helping students achieve the best learning effect, can language learning be more successful.

The two distinctive features of legal English determine that legal English teaching should combine language learning 
with subject knowledge learning, for the purpose of improving students' overall English level and their legal English ability. Therefore, legal English teaching is rather challenging to teachers, who should have good legal knowledge and skillful English proficiency.

\section{Existing Problems in Legal English Teaching}

Legal English course is always welcomed by students; however, there still exist certain problems in terms of setting and teaching.

Firstly, syllabus and curriculum standards are not always unified. With regard to the subject orientation of legal English, different schools take different approaches, some classifying it as professional English while others categorizing it into optional public courses. As a result, the qualifications of teachers, the curriculum system, and teaching methods exhibit great differences.

Secondly, teachers are not well equipped. A sizable proportion of teachers are English majors who have received short-term training of legal knowledge. Professional law teachers who have the background of studying overseas also occupy a substantial percentage. However, those who have dual disciplinary background of law and English account for a small percentage. It is hard for the teachers to form a cooperative teaching team and the teachers' stability and professional identity are not well guaranteed.

Thirdly, the teacher-oriented teaching method is comparatively backward, relying too much on textbooks and courseware. Although such activities as question-and-answer, classroom presentation, and group discussion are often organized in class, the overall teaching, as restricted by class hours and other factors, is still unable to get rid of the concept of authoritative teaching materials and knowledge transmission.

Fourthly, the often used means of evaluation, namely, term papers, assignments, and examinations, fail to effectively reflect the learning process and individual cognitive differences, which will cause the students' lack of initiative of autonomous learning and classroom participation.

In view of the above-mentioned problems, it is of great necessity to innovate, deepen and refine the teaching of legal English. To establish a multimodal mixed teaching mode is a bold but feasible trial.

\section{Multimodality in Legal EngLish Teaching}

\section{A. The Practical Value of Multimodal Legal English Teaching}

The characteristics of legal English determine the uniqueness of its teaching, which is in urgent need of reform. Take Shanghai University of Political Science and Law as an example. As a judicial training base of Shang Cooperation Organization, Shanghai University of Political Science and Law shoulders a great responsibility of cultivating qualified legal talents with international vision, who are familiar with international rules and able to participate in international affairs and competitions. To achieve this glorious mission, foreign language and legal education must be upgraded from being a stepping-stone of promotion and employment to the level of improving foreign language ability and serving the national strategy. The teaching reform and personnel training of foreign language and law should be positioned at this level. The accurate orientation of the course is conducive to the overall development of legal English teaching. In the face of the existing teaching shortcomings, a reform is imperative.

To improve the efficiency of teaching and promote students' internalization of what they have learned, teachers are supposed to use pictures, recordings, videos, film clips, objects, court props and other different modes to organize teaching, giving full play to the synergy and reinforcement between different modes, and organizing teaching in as real a multi-modal situation as possible. In addition, more practical activities such as role playing, court debate, moot court, and court observation should be organized, so as to promote and speed up the transformation of legal English knowledge into legal English ability.

\section{B. Multimodal Teaching as Exemplified by Moot Court}

Moot court has the basic elements and complete process of real court, which is determined by the features of real court. Moot court, according to the degree of authenticity and difficulty, can be categorized into rehearsal form and confrontational form, the former of which is more suitable for the primary stage while the latter of which is for the higher stage. The operation procedures and specific requirements for these two are not identical. Rehearsal moot court refers to the plaintiff and the defendant working together to negotiate the case, define the role, prepare relevant trial materials, and rehearse together, in the hope of completing all the procedures of the moot court. This kind of moot court is similar to a play rehearsal, which requires a planner and organizer who will prepare in advance all the words and deeds of each role and rehearse them repeatedly. The essence of the court trial process is the process of "acting".

From the perspective of multimodality, the trial is a multi-modal symbolic situation (Zhang, 2020). The complete process of the court trial is the one of multimodal synergy, in which different participants in the trial interact with each other in the context of multimodal symbols by virtue of discourse, voice, intonation, action, expression, gesture, posture and other communication modes, find out the facts by means of words, objects, images, audio, video and other media modes, and finally make a decision. Moot court is an important way of legal practical teaching, which has been widely used in legal education. The following will present how a rehearsal moot court can be performed in the legal English course through multimodal means. In the real teaching practice, students can be organized to do the following 
procedures.

Firstly, to select cases and assign roles. It is of great necessity to determine a case with clear disputable points, the complexity and the personnel of which can be set according to the actual needs. Then, separate roles should be assigned, including the director, the judge, the jury, the plaintiff and his lawyer, the defendant and his lawyer, the witness, and the bailiff. There is no requirement for the exact number of the people involved, as the number of lawyers and witnesses is not fixed.

Secondly, to clarify role requirements and analyze the case. Through the analysis of the case, the plaintiff's litigation claim, the cause of action, the required witnesses and evidence, as well as the defendant's defense and the related witnesses and evidence, are all made clear. In addition, the respective roles and tasks of the lawyers, judges, juries and bailiffs of each party are clearly defined, and the trial discourse and relevant materials that each role player needs to prepare are determined through consultation.

Thirdly, to coordinate and rehearse. All the links of the trial and the corresponding tasks of the characters having been clarified, the director and all the participants are supposed to be well-coordinated. During the repeated rehearsals, the script will get modified and polished before finally reaching perfection. Furthermore, necessary props such as judges' robes, mallets, police uniform, and lawyers' uniform need to be well prepared, and the required furnishings of the moot court should be confirmed.

Fourthly, to mock the trial. The whole procedure of the trial is conducted in an orderly manner: the judge announcing the opening of the court session, the opening statement, the court inquiry and debate, the final statement, the jury making a ruling, and the judge pronouncing the sentence.

Through the above steps, we can see that the moot court is presented in a multimodal way, in which different modes interact with each other smoothly and effectively. By simulating the real court environment and trial process, the moot court provides students with opportunities and platforms to apply what they have learned to legal practice.

In terms of confrontational moot court, it resembles real court trial, with high simulation and strong antagonism, in which both parties prepare and rehearse separately, without communicating with each other before the trial. Therefore, it is of high practical value. Similar to rehearsal mock court, confrontational moot court involves setting the case, role division, trial material preparation, case analysis, rehearsal, and formal court session. The differences between confrontational moot court and rehearsal mock court lie in the fact that the former imitates the real trial and emphasizes the substantive confrontation. Therefore, every concrete step should be performed on the spot according to the process of the trial, without any preparation in advance. Confrontational moot court is more demanding and difficult, which requires students to have solid legal English knowledge and high proficiency in listening and speaking. Therefore, this kind of multimodal teaching is suitable for senior students.

\section{Problems to Be Solved in Practice}

To implement the multimodal teaching mode of legal English is challenging. There still remain certain problems to be solved in practice.

\section{A. Contradiction between Multimodal Teaching and Traditional Teaching}

It is noticeable that the multimodal teaching mode of legal English may contradict the traditional teaching mode in that the requirements for time and energy are different. Legal English, as an ESP course, is usually set up as an optional course and covers about two class hours per week. However, the demonstration of multimodal teaching involves a large quantity of time and energy, which puts forward harsh demands for the teacher's competency and devotion. On the other hand, for students, the multimodal teaching mode can be rather helpful for them to grasp in depth certain knowledge points, but it can also be superficial and formal, which is not conducive to the cultivation of their logical thinking ability.

\section{B. The Reform of Ways of Evaluation in Multimodal Teaching Mode}

A comprehensive, objective, real and accurate evaluation system plays a crucial role in realizing the objectives of legal English teaching. Under the multimodal teaching/learning mode, a certain number of students will make best use of the advantages and bypass the disadvantages in terms of evaluation index and mode selection. For instance, while studying the chapter of jury system, those extroverted students tend to choose the mode of dynamic performance to present their learning results, while other students may prefer to study the differences in the jury system between the Anglo-American legal system and the Chinese one. How to evaluate these two groups of students remains a question. In this case, a scientific evaluation system which combines formative evaluation with summative evaluation needs to be established. Students are encouraged to present their studying results in a multimodal way, including self-evaluation, mutual evaluation, group presentation, discussion, and, if necessary, test papers. The performance of each mode is added up to the total score according to a certain proportion. Guided by this kind of evaluation system, students can not only input and output information and knowledge in a multimodal form, but also exercise their critical thinking through traditional reading and writing modes.

\section{Requirements for Teachers'Sustainable Development}


Legal English, if taught in a multimodal mode, will bring about bigger challenges as well more opportunities to teachers. Those teachers who lack the knowledge background of English and American law are supposed to further their study in the hope of becoming qualified legal English teachers. The development of multimodal teaching mode, on the other hand, provides new research directions to the teachers on their teaching methods, classroom case analysis and the transformation of different modes.

Therefore, to satisfy the requirements of multimodal teaching mode for teachers' knowledge and ability, teachers need to keep studying and improve their comprehensive quality.

\section{CONCLUDING REMARKS}

With the rapid development of the rule of law in China, there leaves spacious research space in terms of curriculum, teaching methods and teaching content of legal English, due to the continuous improvement of legal provisions, rich judicial cases, increasing business contacts and diversified legal views. As mentioned at the very beginning, the combination of online and offline hybrid teaching will be the general trend, which is actually a mixture of various modes. Teachers need to make full use of network resources and modern teaching equipment, keeping pace with the times to supplement and update teaching content at any time. Hopefully, the multimodal teaching mode can stimulate students' interest in learning, improve students' ability of autonomous learning and cooperative learning, cultivate students' thinking ability in the interdisciplinary fields of linguistics and law, and enhance their application of legal language and practical ability of foreign legal affairs.

\section{REFERENCES}

[1] Cheng R. L. \& Zhang D. L. (2017). Multimodal Discourse Analysis in China: Taking Journal Articles as an Example. Foreign Languages in China, 7 (3), 36-44.

[2] Gu Y. G. (2007). On Multimedia Learning and Multimodal Learning. Technology Enhanced. Foreign Language Education, (2), 3-12.

[3] Hu Z. L. (2007). Multimodalization in Social Semiotics. Language Teaching and Linguistic Studies, (1), 1-10.

[4] Huang L. H. \& Zhang D. L. (2019). Multi-core Parallel System: Paradigm, Path and Domain Issues of Multimodal Research. Foreign Language Education, (1), 21-26

[5] Jewitt, C., Bezemer, J. \& K., O’Halloran. (2001). Introducing Multimodality. New York: Routledge.

[6] Kress, G. Jewitt, C. Ogborn, J. \& Tsatsarelis. C. (2001). Multimodal Teaching and Learning: The Rhetoric of the Science Classroom. London: Continuum.

[7] Lewis, M. (1985). Practical Techniques for Language Teaching. Hove: Jimmie Hill.

[8] Li Z. Z. (2003). Social Semiotic Approach to Multi-modal Discourse. Foreign Languages Research, (5), 1-8.

[9] Li Z. Z. \& Lu D. Y. (2012). Multimodal Semiotics: Theoretical basis, Research Approaches and Development Prospects. Foreign Languages Research, (5):1-8.

[10] Norris, S. (2011) Identity in (Inter)action: Introducing Multimodal Interaction Analysis. Berlin and New York: Mouton de Gruyter, 39, 41, 48-50.

[11] Qu. W. S. (2017). Legal English Teaching in China: Problems and Prospects. Foreign Languages in China, (4), 4-11.

[12] Royce, T. (2012). Multimodality in the TESOL Classroom: Exploring visual-verbal synergy. TESOL Quarterly, 36 (2): 191-205.

[13] Zhang D. L. (2009). Theoretical Framework for Multimodal Interaction Analysis. Foreign Languages in China, (1), 24-30.

[14] Zhang D. L. (2010). Preliminary Investigation into the Concept of Design and the Selection of Modalities in Multimodal Foreign Language Teaching. Chinese Foreign Languages, (3), 48-53.

[15] Zhang D. L. \& Li Y. X. (2012). A Study of Modal Coordination in Multimodal Classroom Discourse. Foreign Languages and Their Teaching, (1), 39-43.

[16] Zhang F. L. (2019). On the subject orientation of legal English. Foreign Languages in China, (3), 4-9.

[17] Zhang X. (2020). On the Dilemma and Innovative Application of Moot Court in Colleges. Legal System and Society (29), $165-166$.

[18] Zhu Y. S. (2007). Theory and Methodology of Multimodal Discourse Analysis. Foreign Language Research (5), 82-86.

Yuehua Lu was born in Yancheng, Jiangsu, China in 1974. She received her PH. D degree in English Language and Literature from Shanghai International Studies University in 2018. She is currently an associate professor in the School of Languages and Cultures, Shanghai University of Political Science and Law, Shanghai, China. Her research interests include Modern Linguistics, Legal English, and English teaching. 